\title{
Effect of preoperative gabapentin after transurethral prostate resection under general anesthesia
}

\author{
A randomized double-blind, placebo-controlled trial
}

Jinguo Wang, MD, PhD, Guosheng Fu, MD, Junyan Liu, MD, Yunhai Yu, MD, Na Wang, MD, PhD.

\section{ABSTRACT}

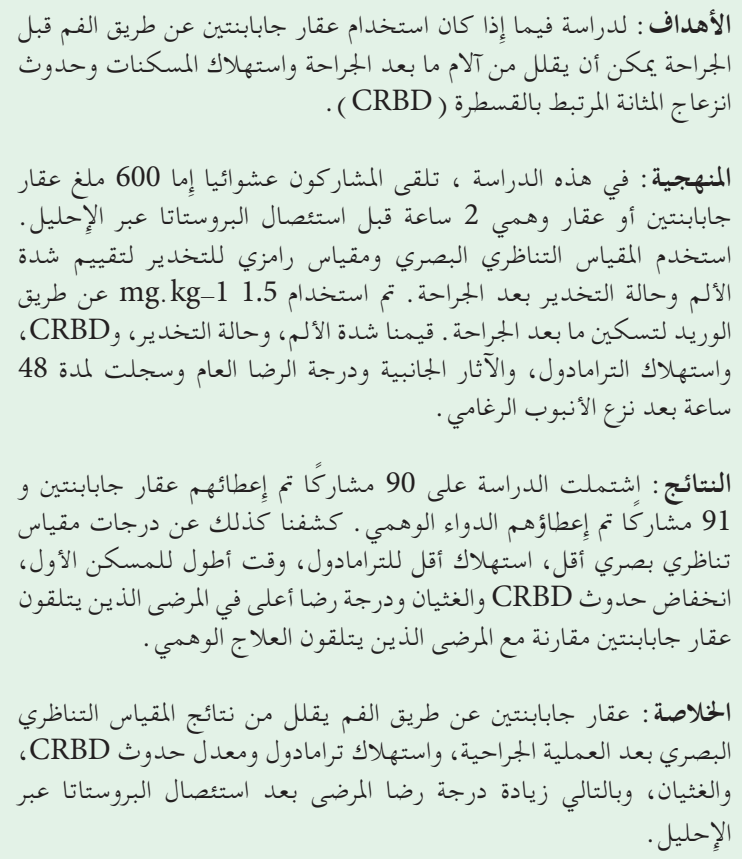

Objectives: To investigate whether preoperative oral gabapentin could reduce postoperative pain, analgesic consumption and the occurrence of catheter-related bladder discomfort (CRBD).

Methods: In this study, participants randomly received either $600 \mathrm{mg}$ gabapentin or placebo orally $2 \mathrm{~h}$ prior to transurethral prostate resection. Visual analogue scale and Ramsay sedation scale was utilized to assess pain intensity and sedation status after surgery. Intravenous $1.5 \mathrm{mg} . \mathrm{kg}-1$ tramadol was used for postoperative analgesia. Pain intensity, sedation status, CRBD, tramadol consumption, side effects and the overall satisfaction degree were assessed and recorded for $48 \mathrm{~h}$ after tracheal extubation.
Results: Ninety participants given gabapentin and 91 participants given placebo completed the study. Lower visual analogue scale scores, less tramadol consumption, longer time to the first analgesic requirement, lower incidence of CRBD and nausea and higher satisfaction degree were detected in the patients receiving gabapentin compared with the patients receiving placebo.

Conclusion: Preoperative oral gabapentin reduced postoperative visual analogue scale scores, tramadol consumption and the occurrence rate of CRBD and nausea, and consequently, increased the degree of patients' satisfaction after transurethral prostate resection.

Keywords: gabapentin, postoperative pain, transurethral prostate resection, catheter-related bladder discomfort

Saudi Med J 2020; Vol. 41 (6): 640-644 doi: 10.15537/smj.2020.6.25132

From the Department of Urology (Wang J, Liu), from the Department of Anesthesiology $(Y u$, Wang $N)$, The First Hospital of Jilin University, Jilin; and from the Department of Urology (Fu), Changling County People's Hospital, Changling, China.

Received 25th March 2020. Accepted 20th May 2020.

Address correspondence and reprint request to: Dr. Na Wang, Department of Anesthesiology, The First Hospital of Jilin University, Changchun, China.E-mail:wangna080613@163.com

ORCID ID: https://orcid.org/0000-0002-8312-9787

$\mathrm{T}$ ransurethral prostate resection (TUPR) can be performed under general, epidural or spinal anesthesia. ${ }^{1-3}$ Patients intend to undergo TUPR under general anesthesia to avoid embarrassment and shame due to lithotomy position during surgery. Transurethral prostate resection is usually considered to be a minor 
surgery with less pain and disability. However, patients undergoing TUPR are often elderly with reduced pain threshold and tolerance. ${ }^{2,3}$ In addition, catheter-related bladder discomfort (CRBD) resulting from catheterrelated bladder irrigation after TUPR is common and very distressing. Therefore, the improvement in pain and discomfort control is necessary.

Multimodal analgesia can decrease opioid consumption and associated adverse effects through synergistic effects. Gabapentin, an anticonvulsant agent, has shown powerful antihyperalgesic proprieties in animal, preclinical and clinical studies. Preoperative administration of gabapentin has been reported to be very effective on postoperative pain relief. ${ }^{4}$ However, the effect of preoperative gabapentin following TUPR has not been evaluated, especially its effect on CRBD due to indwelled urinary catheter and bladder irrigation. The current trial is designed to assess the efficacy of preoperative gabapentin on pain and CRBD following TUPR under general anesthesia.

Methods. This study was designed and conducted according to principles of Helsinki Declaration. Approval from the Institutional Ethic Committee and written informed consent from the participants were obtained. American Society of Anesthesiologists status I or II patients who would have TUPR under general anesthesia were eligible for this clinical trial. Exclusion criteria include allergy to the study medication, renal or hepatic insufficiency, receiving analgesics within 48 hours prior to surgery, chronic pain, drug or alcohol abuse or psychiatric disorder.

The participants were divided in 2 using a sequence of randomization numbers generated by a computer. A physician who was not a member of this study prepared the envelopes containing the study drugs. The patients orally received $600 \mathrm{mg}$ gabapentin (Nhwa, Jiangsu Nhwa Pharmaceutical Co., Ltd., Xuzhou, China) or placebo with sips of water 2 hours prior to operation.

General anesthesia using a laryngeal mask airway was performed in all patients without premedication. After anesthesia induction using $0.3 \mathrm{mg} \cdot \mathrm{kg}^{-1}$ etomidate, 3 $\mu \mathrm{g} . \mathrm{kg}^{-1}$ fentanyl, and $0.15 \mathrm{mg} . \mathrm{kg}^{-1}$ cisatracurium, propofol $\left(6\right.$ to $\left.8 \mathrm{mg} \cdot \mathrm{kg}^{-1} \cdot \mathrm{h}^{-1}\right)$ and remifentanil $\left(0.012 \mathrm{mg} \cdot \mathrm{kg}^{-1} \cdot \mathrm{h}^{-1}\right)$ were used to keep BIS between 45 and 60 during the

Disclosure. Authors have no conflict of interests, and the work was not supported or funded by any drug company. operation. Ondansetron $4 \mathrm{mg}$ and flurbiprofen axetil $1 \mathrm{mg} \cdot \mathrm{kg}^{-1}$ were given intravenously when the surgery was completed. The participants were extubated when they got their adequate spontaneous respiration back and responded to verbal commands. After surgery, all patients had bladder irrigation according to standard prescription of the department.

A physician who was not a member of the study assessed pain intensity at 2, 4, 8, 16, 24, 36 and 48 hours after tracheal extubation with visual analogue scale (VAS) $(0=$ indicated no pain and $10=$ indicated worst pain ever). At the same time points, the level of sedation was assessed using Ramsay sedation scale (RSS) ( 1 = anxious and agitated; 2 = cooperative and tranquil; 3 = drowsy but responded to command; 4 = asleep but responded to tactile stimulation; and $5=$ no response).

When VAS score was more than 3, the patient received $1.5 \mathrm{mg} \cdot \mathrm{kg}^{-1}$ tramadol by the intravenous route. Time to the first analgesic demand and analgesic consumption were collected. If a patient complained of any of these symptoms of CRBD, including urethral discomfort, urgent urination and discomfort in the suprapubic region, $5 \mathrm{mg}$ solifenacin succinate was given by the oral route. Associated adverse effects were observed for 48 hours. Over sedation is defined as RSS score equal to 5 . Oxygen saturation less than $90 \%$ on air was taken as respiratory depression. The degree of overall satisfaction was assessed with poor, moderate, good, and excellent.

Data analyses were conducted utilizing SPSS Statistics for Windows, version 17.0 (SPSS Inc., Chicago, Ill., USA). The primary endpoint was postoperative VAS score. A minimum difference of one VAS score is considered to have clinical significance. The sample size of at least 90 patients each group was obtained with 2 -sided significance of $5 \%$ and power of 90\%. We enrolled 99 participants per group. A $p<0.05$ was considered significant. Normally distributed data were processed using unpaired t-test, VAS and RSS scores using Mann-Whitney U-test, and categorical variables utilizing $x^{2}$ or Fisher's exact test.

Results. At first, 198 patients were qualified for this clinical trial, but only 181 accomplished this study. Fourteen patients were excluded, because of refusal to participate in this study. After randomization, 3 patients were removed from the statistical analysis due to refractory postoperative fever (one patient in each group) and postoperative bleeding (one patient in the gabapentin group).

Table 1 showed that no difference in demographic and surgical data was found between the 2 groups. The 
gabapentin group had significantly longer time to the first analgesic demand, fewer participants who required postoperative analgesia and fewer doses of tramadol administrated than the control group (Table 2).

Visual analogue scale scores at 2, 4, 8 and 16 hours following tracheal extubation were lower in the gabapentin group compared with the control group (Figure 1). Ramsay sedation scale scores were similar at all measured time points between 2 groups (Figure 2). The gabapentin group had lower occurrence rates of CRBD and nausea than the control group.

Table 1 - Patient demographics and surgical data.

\begin{tabular}{lccc}
\hline $\begin{array}{l}\text { Patient's } \\
\text { demographics }\end{array}$ & $\begin{array}{c}\text { Gabapentin } \\
\text { group } \\
(\mathbf{n}=90)\end{array}$ & $\begin{array}{c}\text { Control } \\
\text { group } \\
(\mathbf{n}=91)\end{array}$ & $P$-value \\
\hline Age (year) & $66.9 \pm 9.5$ & $68.1 \pm 8.8$ & 0.379 \\
Weight (kg) & $58.7 \pm 9.2$ & $60.3 \pm 11.1$ & 0.292 \\
Height (cm) & $168.9 \pm 8.7$ & $170.1 \pm 9.5$ & 0.376 \\
ASAI/II (n) & $68 / 22$ & $59 / 32$ & 0.143 \\
$\begin{array}{l}\text { Duration of surgery } \\
\text { (min) }\end{array}$ & $56.8 \pm 12.3$ & $59.7 \pm 19.7$ & 0.237 \\
$\begin{array}{l}\text { Prostate volume (g) } \\
\text { Duration of } \\
\text { anesthesia (min) }\end{array}$ & $58.8 \pm 11.8$ & $61.7 \pm 12.7$ & 0.113 \\
\hline
\end{tabular}

Values are presented as mean \pm standard deviation and number of patients. ASA: American Society of Anesthesiologists.
Five patients experienced decreased oxygen saturation. They responded promptly to increased oxygen rate and arousing, and needed no further intervention to maintain their oxygen saturation at $95 \%$ or higher. No respiratory depression and over sedation were observed (Table 3).

Table 4 showed that the gabapentin group had a higher degree of overall satisfaction than the control group.

Discussion. This study demonstrates that preoperative gabapentin can reduce postoperative VAS

Table 2 - Postoperative tramadol use.

\begin{tabular}{lccc}
\hline Tramadol use & $\begin{array}{c}\text { Gabapentin } \\
\text { group } \\
(\mathbf{n}=90)\end{array}$ & $\begin{array}{c}\text { Control } \\
\text { group } \\
(\mathbf{n}=91)\end{array}$ & $P$-value \\
\hline $\begin{array}{l}\text { Time to the first tramadol } \\
\text { request (min) }\end{array}$ & $123.8 \pm 49.7$ & $101.6 \pm 41.8$ & 0.0014 \\
$\begin{array}{l}\text { Patients requiring tramadol } \\
\text { analgesia: (\%) }\end{array}$ & $57(63.3)$ & $72(79.1)$ & 0.029 \\
$\begin{array}{l}\text { Doses of tramadol received }(n) \\
0\end{array}$ & 33 & 19 & \\
1 & 33 & 35 & \\
2 & 22 & 29 & \\
3 & 2 & 8 & \\
\hline
\end{tabular}

Values are presented as mean \pm standard deviation and number of patients (n).

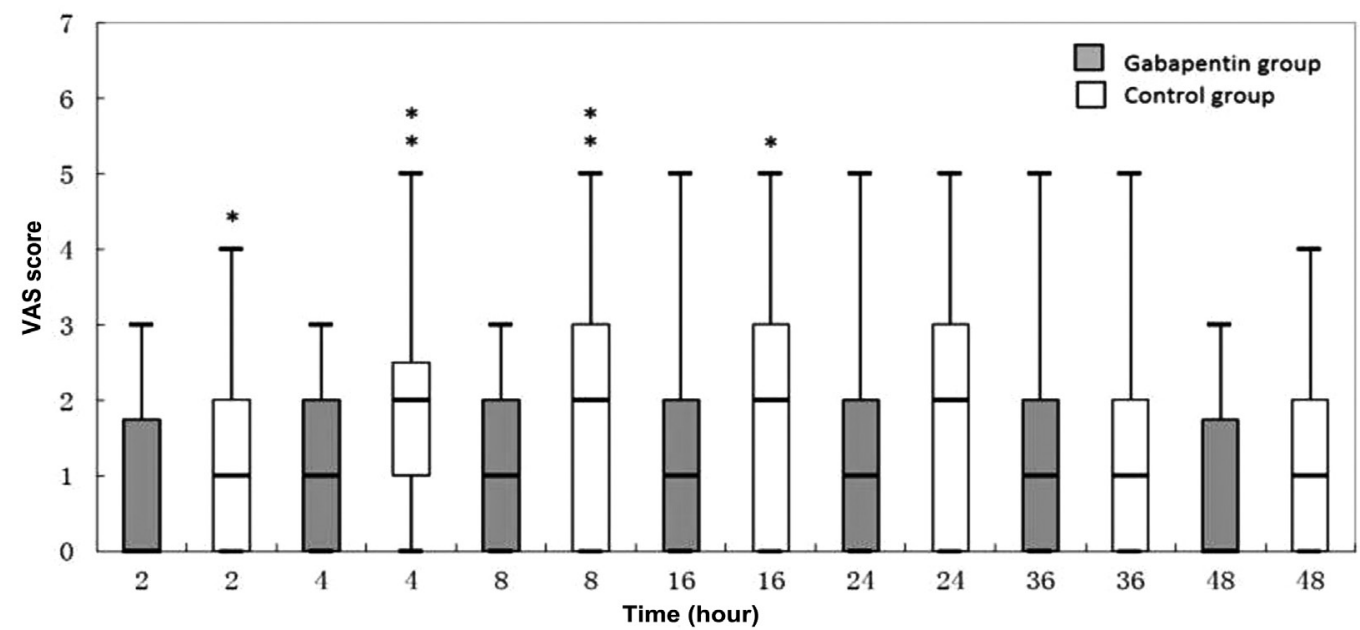

Figure 1 - Visual analog scale (VAS) scores at various time points after tracheal extubation. Box plots of postoperative VAS scores. Results are expressed in median. The top and bottom of each box indicate 75 th and 25 th percentiles and the error bars maximum and minimum. ${ }^{*}$ indicates $p<0.05$ compared with the control group, ${ }^{*}$ indicates $p<0.01$ compared with the control group. 


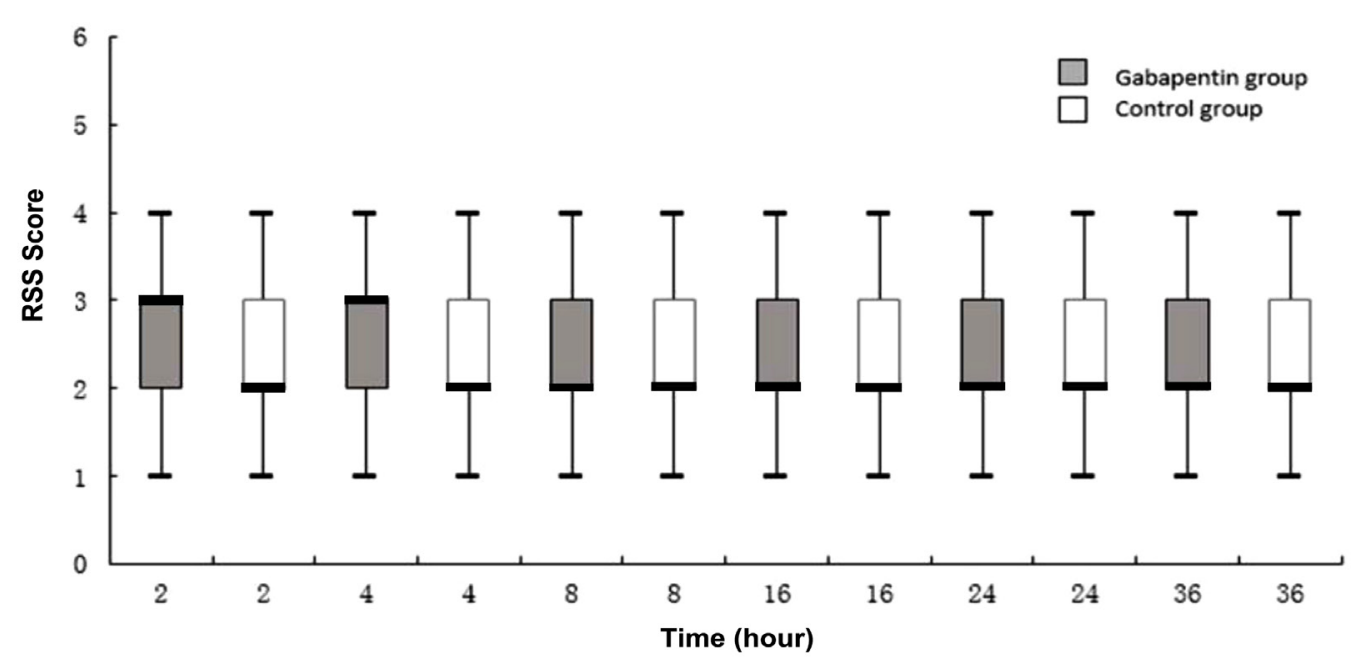

Figure 2 - Ramsay sedation scale (RSS) scores at various time points postoperatively. Box plots of postoperative RSS scores. Results are expressed in median. The top and bottom of each box indicate 75 th and 25 th percentiles and the error bars maximum and minimum.

Table 3 - The incidence of catheter-related bladder discomfort (CRBD) and side effects.

\begin{tabular}{|c|c|c|c|}
\hline Side effects & $\begin{array}{c}\text { Gabapentin } \\
\text { group } \\
(\mathrm{n}=90)\end{array}$ & $\begin{array}{c}\text { Control } \\
\text { group } \\
(n=91)\end{array}$ & $P$-value \\
\hline CRBD & $58(64.4)$ & $74(81.3)$ & 0.017 \\
\hline Decreased oxygen saturation & $4(4.4)$ & $1 \quad(1.1)$ & 0.210 \\
\hline Over sedation & $0(0)$ & $\begin{array}{ll}0 & (0)\end{array}$ & - \\
\hline Nausea & $10(11.1)$ & $18(19.8)$ & 0.159 \\
\hline Vomiting & $2(2.2)$ & $4 \quad(4.4)$ & 0.688 \\
\hline Headache & $2(2.2)$ & $5(5.5)$ & 0.443 \\
\hline Dizziness & $6 \quad(6.7)$ & $11(12.1)$ & 0.319 \\
\hline Pruritis & $3(3.3)$ & $5(5.5)$ & 0.729 \\
\hline
\end{tabular}

Values are presented as number of patients and percentage (\%). CRBD: catheter-related bladder discomfort.

Table 4 - Patient satisfaction.

\begin{tabular}{lcc}
\hline $\begin{array}{l}\text { Patient's } \\
\text { satisfactions }\end{array}$ & $\begin{array}{c}\text { Gabapentin } \\
\text { group } \\
(\mathbf{n}=90)\end{array}$ & $\begin{array}{c}\text { Control } \\
\text { group } \\
(\mathbf{n}=91)\end{array}$ \\
\hline Poor & $1(1.1)$ & $4(4.4)$ \\
Moderate & $14(15.6)$ & $23(25.3)$ \\
Good & $32(35.6)$ & $38(41.8)$ \\
Excellent & $43(47.8)$ & $26(28.6)$ \\
\hline
\end{tabular}

Values are presented as number of patients and percentage (\%). $p=0.0338$ score, tramadol consumption and incidence of CRBD and nausea. Decrease in VAS score definitely means better pain control. Many published randomized studies indicate that preoperative gabapentin has a useful effect on postoperative pain relief and analgesic comsumption after various surgeries. ${ }^{4}$ Various meta-analyses and systematic reviews report that preoperative gabapentin decreases pain intensity and analgesic demand on the first postoperative day. ${ }^{4-6}$ The rationale behind analgesic effect of preoperative gabapentin is that antinociceptive treatment started before the surgical stimuli is more efficient in decreasing postoperative pain than treatment initiated in the early postoperative period, as it can reduce hyper sensitivity induced by the surgical procedures in central nerve system. ${ }^{7}$

Gabapentin can easily attach to $\alpha-2-\delta$ subunits of presynaptic voltage-gated calcium channels. Thereafter, calcium influx is hindered, leading to excitatory neurotransmitters release involved in central sensitization. ${ }^{8}$ Gabapentin has demonstrated the evidence of antihyperalgesic benefit, because it is likely to prevent the development of central sensitization. In addition, gabapentin can produce synergistic action when combined with other analgesics, for example tramadol. ${ }^{9}$ Therefore, preoperative gabapentin is especially beneficial in acute postoperative pain.

The catheter-related bladder irritation afterTUPR can lead to CRBD immediately after surgery, especially when the patient is catheterized under anesthesia. It is reported that gabapentin is effective in prevention of CRBD after percutaneous nephrolithotomy. Transurethral prostate resection leads to local physiological changes 
immediately after surgery, so it is necessary to investigate the effect of preoperative gabapentin on CRBD after TUPR. It is hypothesized that bladder C-fiber afferent nerve function is upregulated in CRBD. ${ }^{10}$ Gabapentin may inhibit activity of the afferent C-fibers, and then prevent peripheral sensitization. ${ }^{11}$ Gabapentin is also effective in neurogenic over-activity, because it can modulate irritability of the sacral reflex center. ${ }^{12}$ The combined peripheral and central action of gabapentin may contribute to decrease in the occurrence of CRBD.

Contrary to our results, there are studies which are unable to display a decrease in pain scores and analgesic requirement following surgery in patients administrated preoperative gabapentin. ${ }^{13}$ The different findings may result from the different types of surgeries or smaller dose of gabapentin administrated.

The doses between 300 and $1200 \mathrm{mg}$ of gabapentin have been used for preoperative administration and well tolerated orally. ${ }^{4-6}$ The dose of $600 \mathrm{mg}$ has been found to be a proper dose for preoperative use. Larger dose may increase the occurrence of side effects, and smaller dose is likely related with inadequate effect. Two hours prior to surgery is probably an optimal time of administration because it consists with maximum plasma concentration.

Gabapentin do not influence hemodynamics intraoperatively and the safety profile is especially beneficial to the elder patients. ${ }^{4}$ Sedation and dizziness are the common side effects of gabapentin. This study demonstrates that preoperative gabapentin has no significant effect on postoperative sedation status (RSS score) or anesthetic recovery (duration of anesthesia). This study also displays significantly decreased incidence of nausea, which may result from the tramadol sparing effect. The result is consistent with previous reports. ${ }^{6}$

Study limitations. Only total consumption of tramadol for 48 hours is recorded, so our results cannot demonstrate if the analgesic sparing efficacy of gabapentin extends beyond the study period. The other limitation of this clinical trial is that our results reveal that preoperative gabapentin decrease VAS scores only in the first postoperative day, but unable to display the exact time period of the tramadol sparing effct as we only recorded the time tramadol was needed.

In conclusion, preoperative gabapentin reduces postoperative pain intensity, tramadol consumption and occurence of CRBD and nausea, and consequently, improves patients' satisfaction after TUPR.

Acknowledgment. We would like to thank Jiahe Medical English Editing Company for English language editing.

\section{References}

1. Wang J, Pang L, Han W, Wang N. Effect of preemptive intravenous oxycodone on low-dose bupivacaine spinal anesthesia with intrathecal sufentanil. Saudi Med J 2015; 36: 437-441.

2. Li H, Li Y, He R. Sparing effects of sufentanil on epidural ropivacaine in elderly patients undergoing transurethral resection of prostate surgery. Yonsei Med J 2015; 56: 832-837.

3. Wang J, Fu Y, Ma H, Wang N. Effect of Preoperative intravenous oxycodone after transurethral resection of prostate under general anesthesia. Int Surg 2017; 102: 377-381.

4. Mao Y, Wu L, Ding W. The efficacy of preoperative administration of gabapentin/pregabalin in improving pain after total hip arthroplasty: a meta-analysis. BMC Musculoskelet Disord 2016; 17: 373.

5. Zhai L, Song Z, Liu K. The Effect of Gabapentin on Acute Postoperative Pain in Patients Undergoing Total Knee Arthroplasty: A Meta-Analysis. Medicine (Baltimore) 2016; 95 : e3673.

6. Li S, Guo J, Li F, Yang Z, Wang S, Qin C. Pregabalin can decrease acute pain and morphine consumption in laparoscopic cholecystectomy patients: A meta-analysis of randomized controlled trials. Medicine (Baltimore) 2017; 96: e6982.

7. Penprase B, Brunetto E, Dahmani E, Forthoffer JJ, Kapoor S. The efficacy of preemptive analgesia for postoperative pain control: a systematic review of the literature. AORN J 2015; 101: 94-105.e8.

8. Shakshuki A, Agu RU. Compounded topical gabapentin for neuropathic pain: Does choice of base affect efficacy? Int $J$ Pharm Compd 2019; 23: 496-503.

9. Chang CY, Challa CK, Shah J, Eloy JD. Gabapentin in acute postoperative pain management. Biomed Res Int 2014; 2014: 631756.

10. Lee C, Chermansky CJ, Damaser MS. Translational approaches to the treatment of benign urologic conditions in elderly women. Curr Opin Urol 2016; 26: 184-192.

11. Chua ME, See MC 4th, Esmeňa EB, Balingit JC, Morales ML Jr. Efficacy and safety of gabapentin in comparison to solifenacin succinate in adult overactive bladder treatment. Low Urin Tract Symptoms 2018; 10: 135-142.

12. Pannek J. Overactive bladder-which treatment when? Urologe A 2017; 56: 1532-1538.

13. Ahearn B, Kumar A, Premkumar A, Samady H, Gottschalk M, Xerogeanes J, et al. Effect of preoperative gabapentin with a concomitant adductor canal block on pain and opioid usage after anterior cruciate ligament reconstruction. Orthop J Sports Med 2019; 7: 2325967119828357. 